\title{
Automatic Washing Machine using PLC
}

\author{
V.V.S.Madhuri \\ Assistant Professor, EEE \\ GRIET \\ Hyderabad, Telangana-India \\ Naveen Yerrathota \\ Dept. of EEE, GRIET \\ Hyderabad, Telangana - India.
}

\begin{abstract}
This paper presents a design of automated washing machine which is controlled using programmable logic controller. Programmable logic controller (PLC) was used to control a mechatronics system using specific functions. Basic PLC functioning like timing, sequencing, controlling and relaying were implemented. The hardware contains one agitator and shell tub. A split phase induction motor is used to run the agitator in either direction required as per the sequence programmed. Solenoid valves are used for water inlet and drain out. Operation of these devices (motor and solenoid valves) is completely automated using PLC.
\end{abstract}

Keywords - PLC, Ladder Programming, Split Phase Induction motor, Solenoid Valves.

\section{INTRODUCTION}

A washing machine is an automatic machine, controlled by a motor and different hardware equipment, whose operation is sequenced and automated without human interfernce. This term is mostly applicable only for the machines which are operating with water as the primary cleaning solution, which is completely opposed to some other technologies which works under dry cleaners as well ultrasonic cleaners.

Washing entails immersing,dipping,rubbing or scrubbing in water or other liquids, which is usually accompanied by soap ,detergent and bleach which is active ingredient with $\mathrm{NaClO}$. Improvement in technology ,search for higher integration, invention of new ideas, and development in the world of electronics has brought about an increased demand for the automation control for most machinery and their processes.

There are two electronic controllers which are analog and digital controllers. Analog controllers uses basic forms of operational amplifier circuits or other forms of control actions. The digital contollers are microprocessor \& microcontroller based system like PLC

Millenium Crouzet PLC is used for the automation of Washing machine. A sequence of operations like Washing Rinsing - Squeezing - and Drying are programmed in Ladder Programming.

\section{PROGRAMMABLE LOGIC CONTROLLER (PLC):}

PLCs are miniature computers that control the automated systems to run the everything around us .It controls the equipment in real time according to pre-programmed set of instructions. Now a day's technology is totally followed in integration of IOT, it is most advantage to monitor operation, or making some changes in $\mathrm{I} / \mathrm{O}$ connections through our smart phones .

PLC is an electronic device, in past it is pronounced as "sequence controller".

PLC reads the status of the external input devices, such as keypad, sensor, switch and pulses, and execute by the microprocessor logic, sequential, timing, counting and arithmetic operations according the

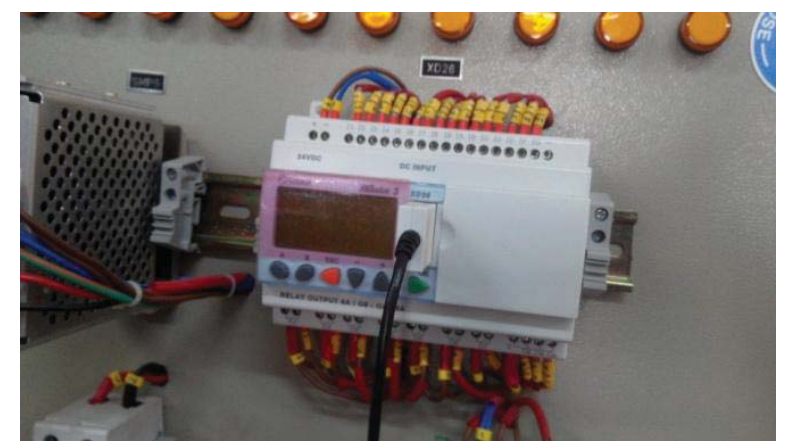


status of the input signals as well as the pre-written program stored in the PLC. The input/output signals of PLC include DI (digital input), AI (analog input), PI (pulse input), NI (numeric input), DO (digital output), AO (analog output), and PO (pulse output). Hence,PLC will still evergreen in the industrial automation field in the future.

The mostly used language in designing a PLC program are Ladder Diagram(LD), Functional Block Diagram (FBD).

\section{Hardware:}

PLC hardware refers to the individual PLCs that monitor equipment. Allen Bradley (AB), Automation Direct PLC, Siemens, Square D and Omron are some of the major PLC hardware providers of PLC hardware.

Some PLC hardware such as the Click series Automation Direct PLC can be combined to create an operator interface. Other PLC devices require connective devices to communicate with an operator interface or networking device.

Software :

a) PLC automation software is usually written in the form of a ladder program. The ladder program is stored in a flash EEPROM memory inside the PLC.

b) PLC ladder logic programming software itself typically runs on a PC where the ladder logic code is created by a programmer.

c) Operator interface software is typically Microsoft Windows based. For example, Automation Direct PLC C-More touch panel programming software runs on any Windows OS. CoDeSys, short for Controller Development System, is open source PLC software. TRiLOGI is a ladder logic and BASIC based programming language, though it is limited to only a few series of PLCs. Automation Direct's DataWorx software and NI LabVIEW software are two of the major providers of data logging software.

\section{SOFTWARE DEVELOPMENT}

The Software used for this project is MILLENIUM 3 Programming Language. The programming language used is Ladder Logic programming which includes Timers and Counters and specific Functions such as Time Management, Character String, Communication, etc

\section{A Edit mode:}

The Edit mode is used to construct program, which corresponds to the development of the application. In this mode you can: create a password, protect your program, display dependencies between blocks, display a parameter summary table, preview function blocks by theme, or obtain online help for each function block.

\section{B Simulation mode.}

When in Simulation mode the program is executed offline directly in the programming . Changing the state of an input, output forcing updates the simulation windows. You can simulate a power failure or program timing, modify analog variables via the Millenium's screen, or create a time-based jump even without changing the time on the PC.

\section{Monitoring mode}

When in Monitoring mode the program is executed on the controller and the programming workshop is connected to the controller. In this mode you can view machine operation in real time on your PC, you can change the parameters via the front panel, or conduct progressive debugging and validate each part of the application.

In simulation and monitoring modes, it is possible to:

1. view the input and output functions in the supervision window which is user friendly

2. give the inputs or outputs to test program behavior under specific conditions.

\section{MILLENIUM-3}

The MILLENIUM 3 is programmed using the CLS M3software workshop. It should therefore be connected to your PC. Editing a program from the Millenium front panel is not possible.

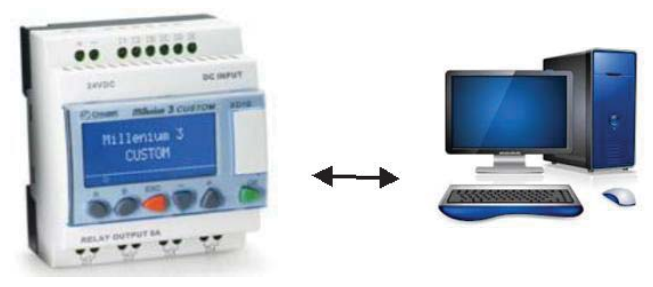




\section{PC resources:}

Get started with PC Pentium II300 MHz (600 MHz is recommended), 128Mb of RAM memory (256 MB recommended) and Compatible with windows 2000, NT 4.0 SP5, XP, Vista and Windows.

\section{ALGORITHM}

1) Inlet valve for water is on for 10 seconds and then off for remaining time. Motor starts running after 10 seconds.

2) Motor Running

Forward direction: 5 seconds

Stop time: $\quad 1$ second

Reverse direction: 8 seconds

Stop time: $\quad 1$ second

This occurs for 2 cycles.

Motor stops running

3) Then output valve remains open for whole period.

4) Motor starts running after 5 seconds

Motor Running

Forward direction: 5 seconds

Stop time: $\quad 1$ second

Reverse direction: 8 seconds

Stop time: $\quad 1$ second

This occurs for 1 cycle.

Motor stops running.

5) Output valve stops at end after 10- 15 seconds.

\section{Screenshot of program:}

\section{SOFTWARE PROCEDURE:}

1. Draw the ladder diagram

2. Configure the connection for target peripheral to react that is microcontroller.

3. Then Write to the controller (program is fed into the controller) and tap OK for the next steps.

4. Tap on the Monitoring (M) mode for real time hardware control.

5. Then tap ON with reset button to start the program.
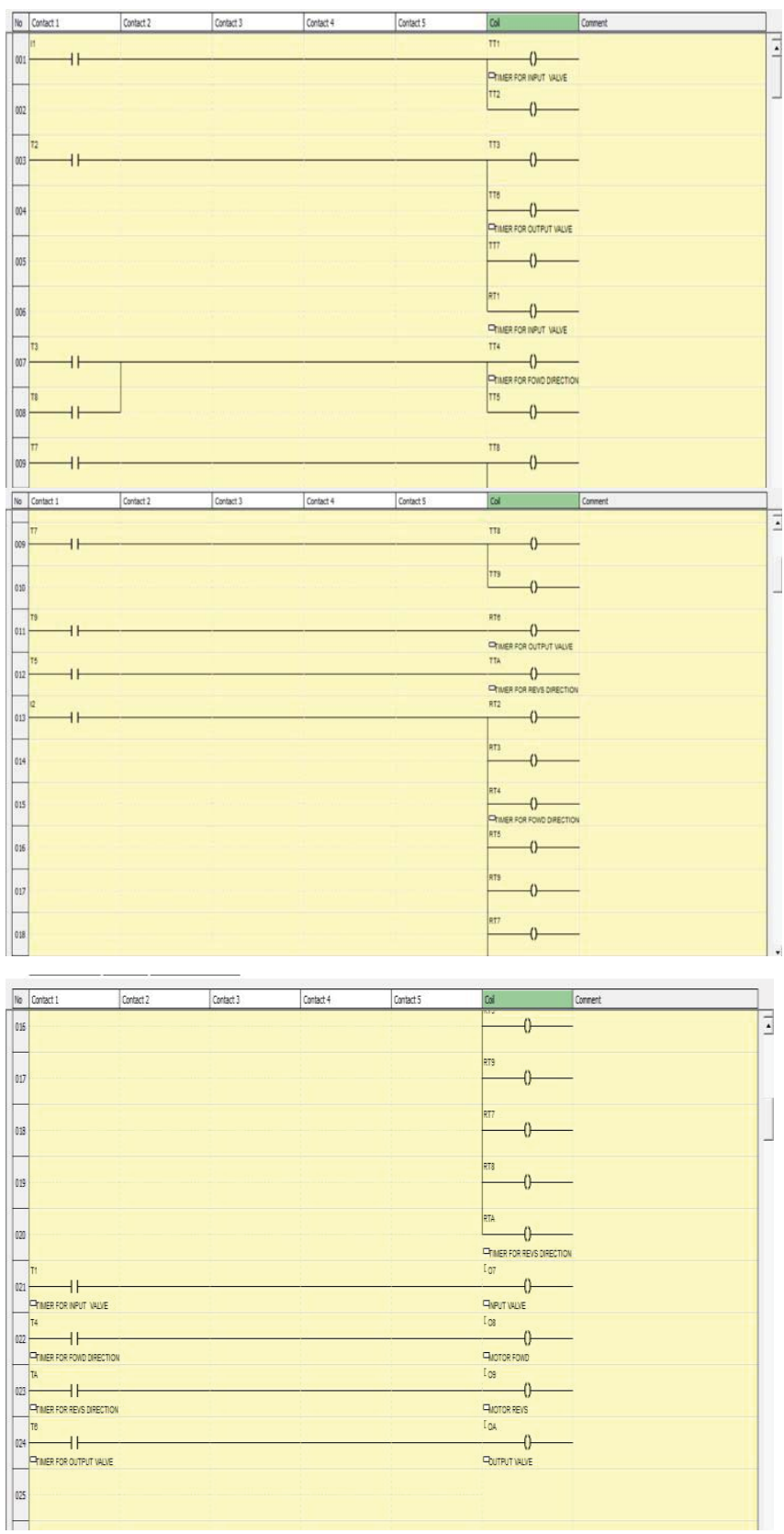


\section{HARDWARE IMPLEMENTATION:}

The hardware contains one agitator and shell tub. A split phase induction motor is used to run the agitator in either direction required as per the sequence programmed. Solenoid valves are used for water inlet and drain out. Operation of these devices (motor and solenoid valves) is completely automated using PLC as shown in the Algorithm.

When supply is on, initially the inlet valve is on and water is filled into the agitator. After a set time delay, the induction motor starts the agitator rotates in clockwise and anti-clockwise direction with certain delay. The motor stops, the outlet valve is on so the water drains out. The motor again starts only in one direction and stops after a preset time delay.

\section{INDUCTION MOTOR}

An induction or Asynchronous motor is an AC electric motor in which the electric current in the rotor needed to produce torque is obtained by electromagnetic induction from the magnetic field of the stator winding.

An induction motor can therefore be made without electrical connections to the rotor as are found in universal, DC and synchronous motors.

Split phase Induction motor is used for the present application. Larger single phase motors are split-phase motors and have a second stator winding fed with out-of-phase current; such currents may be created by feeding the winding through a capacitor or having it receive different values of inductance and resistance from the main winding.

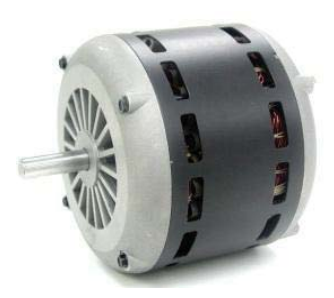

\section{Emerson Commander SK AC Drive:}

The VFD drive used is a product manufactured by Emerson. The figure shown below is Emerson commander sk ac drive. This is the AC drive used for controlling the speed of the 3-phase induction motor. In this drive we can adjust the voltage and frequency at a time, so the speed of the 3-phase induction motor is increased or decreased according to the values given. The Commander SK is an open loop vector $\mathrm{AC}$ variable speed inverter drive used to control the speed of an AC induction motor. The drive uses an open loop vector control strategy to maintain almost constant flux in the motor by dynamically adjusting the motor voltage according to the load on the motor. The AC supply is rectified through a bridge rectifier and then smoothed across high voltage capacitors to produce a constant voltage DC bus. The DC bus is then switched

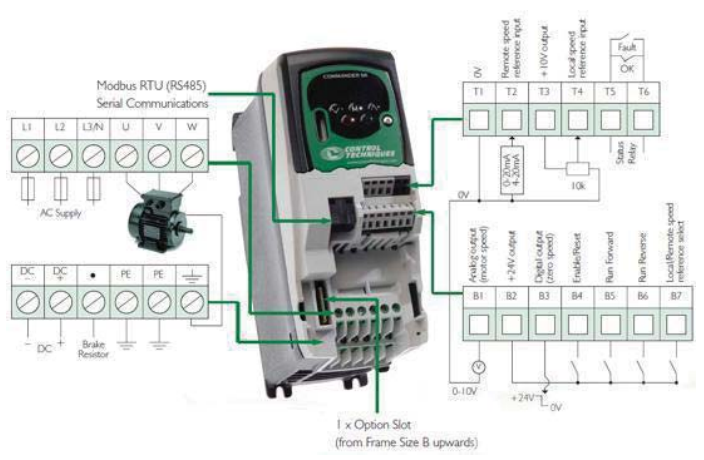

through an IGBT bridge to produce $\mathrm{AC}$ at a variable voltage and a variable frequency. This $\mathrm{AC}$ output is synthesized by a pattern of on-off switching applied to the gates of the IGBTs. This method of switching the IGBTs is known as Pulse Width Modulation (PWM).

Specifications:

The specifications of V/F AC drive are: Model No.: SKA1200075

Voltage : (200-240)V Current: 10.5A Frequency: $50-60 \mathrm{~Hz}$

Driving capability: 3 -phase $0-240 \mathrm{~V}$ $0-1500 \mathrm{rpm}$

$4 \mathrm{~A}$

Description:

The V/F drive being used has 9 different configurations i.e., the $\mathrm{V} / \mathrm{F}$ drive can be operated in 9 different modes. Each mode or configuration has some pre-defined operation. There are three physical sizes comprising 15 different models. The input voltage ranges are

\begin{tabular}{|c|l|}
\hline Configuration & \multicolumn{1}{|c|}{ Description } \\
\hline Al.AV & Voltage and current input \\
\hline AV.Pr & Voltage input and 3 preset speeds \\
\hline Al.Pr & Current input and 3 preset speeds \\
\hline Pr & 4 preset speeds \\
\hline PAd & Keypad control \\
\hline E.Pot & Electronic motorized potentiometer control \\
\hline tor & Torque control operation \\
\hline Pid & PID control \\
\hline HVAC & Fan and pump control \\
\hline
\end{tabular}


single phase input, 200 to $240 \mathrm{~V}$,

three phase input, 200 to $240 \mathrm{~V}$, three phase input

380 to $480 \mathrm{~V}$ and are power dependent.

The 9 different configurations are

Voltage Preset Mode:

Each mode has a pre-defined operation. The mode we are using in this project is AV.PR (voltage preset). In this mode three speeds can be preset. The figure below shows the basic terminals of the AC drive in AV.PR mode. Using this $\mathrm{V} / \mathrm{F}$ drive not only the speed but also the direction of rotation of the motor can be controlled. The switches B4,B5,B6 denotes enable, run forward and run reverse. The motor rotates in forward or clockwise direction when both the switches B4 and B5 are closed or are in ON position. The motor rotates in reverse direction or anticlockwise direction when the switches B4 and B6 are closed or in ON position. The switch B4 must be always ON or in closed position for ant operation to take place. The drive consists of switches name T1-T4 and B1-B7. The connections are made according to the one as shown in the fig below for the drive to operate in Av.PR mode. By operating or connecting the switches $\mathrm{T} 4$ and B7 as shown in the table below, the speed of the induction motor can be set to a preset value.

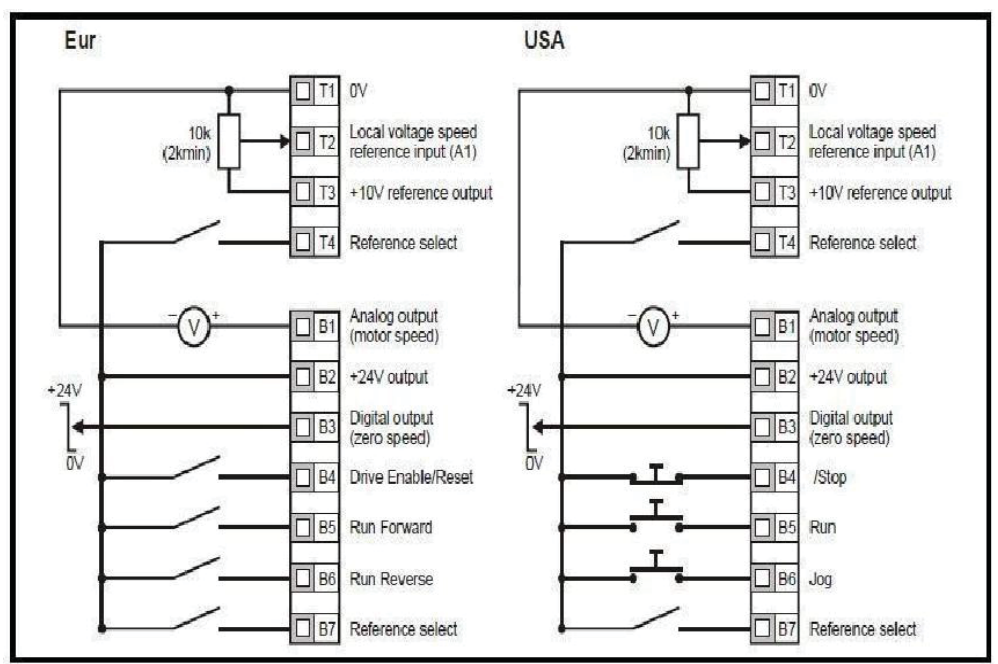

\begin{tabular}{|c|c|c|}
\hline $\mathrm{T} 4$ & $\mathrm{~B} 7$ & Reference selected \\
\hline 0 & 0 & $\mathrm{~A} 1$ \\
\hline 0 & 1 & Preset 2 \\
\hline 1 & 0 & Preset 3 \\
\hline 1 & 1 & Preset 4 \\
\hline
\end{tabular}

VII. SOLENOID VALVES:

A solenoid valve is an electromechanically operated valve. The valve is controlled by an electric current through a solenoid: in the case of a two-port valve the flow is switched on or off; in the case of a three-port valve, the outflow is switched between the two outlet ports. Multiple solenoid valves can be placed together on a manifold.

Solenoid valves are the most frequently used control elements in fluidics. Their tasks are to shut off, release, dose, distribute or mix fluids. Solenoids offer fast and safe switching, high reliability, long service life, good medium compatibility of the materials used, low control power and compact design.

Direct Current Or Alternating Current Powered solenoid valve is used for the present application.

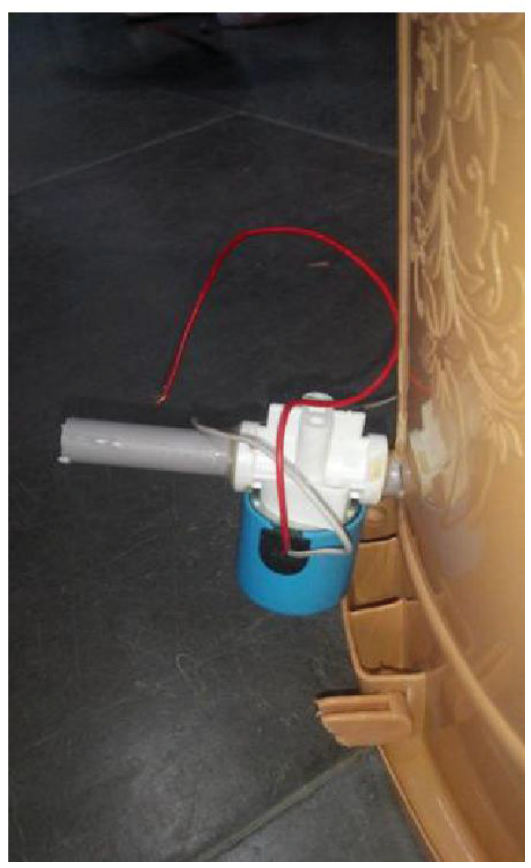




\section{RESULTS}

This project is done to automate a model of washing machine by controlling the inlet, outlet \& rotating mechanism in washing machine by using PLC which is the future and present technology which is widely used in industrial and home automation

The integration of PLC technology for home automation devices allows for end-to-end remote monitoring, control and surveillance reaching places where no cabling exists other than power wires.
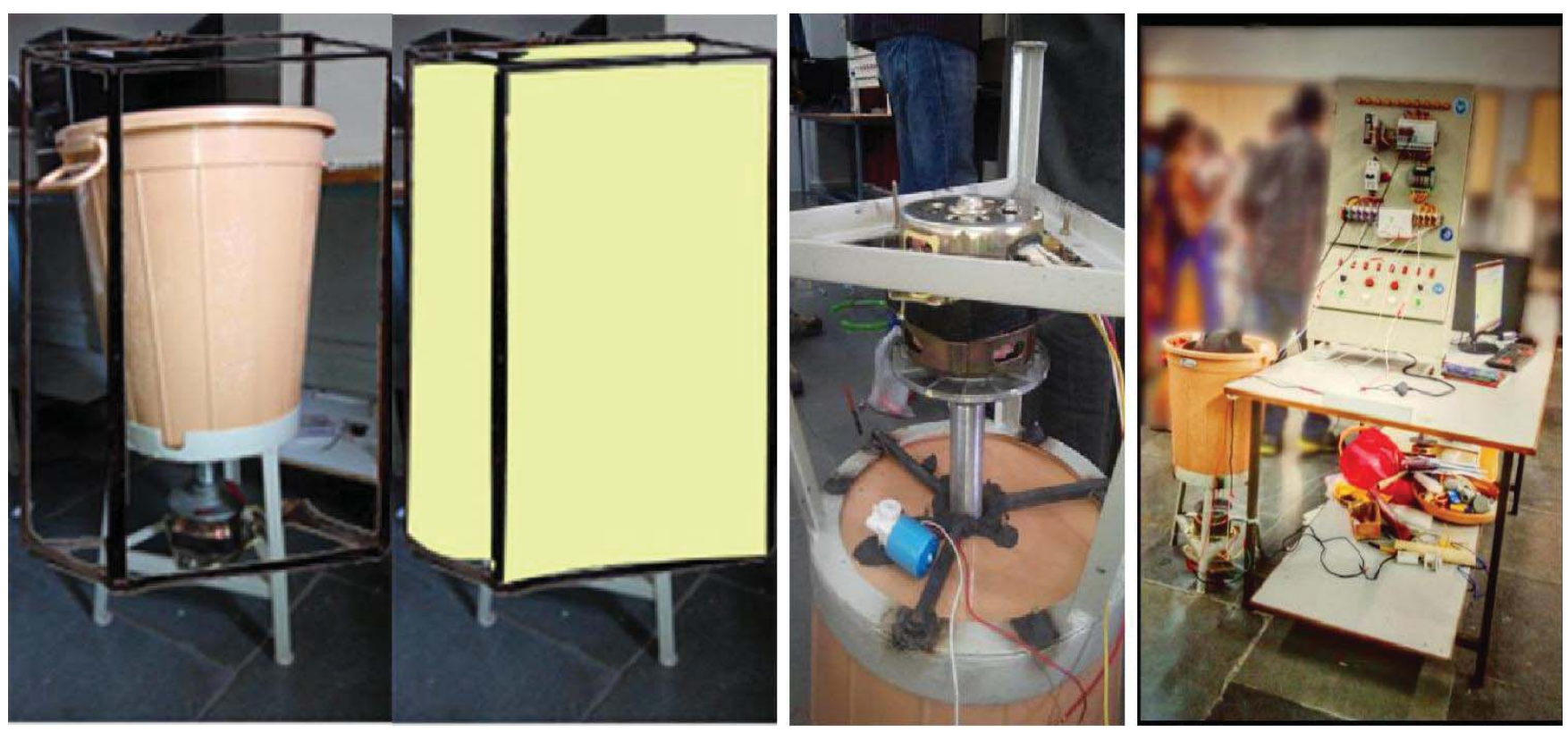

The above pictures show the complete setup of demonstration of a PLC based Automatic Washing Machine.

\section{REFERENCES}

[1] https://en.wikipedia.org/wiki/Washing machine

[2] http://www.circuitsgallery.com/2012/11/what-is-PLC.html

[3] http://www.easyveep.com/modules.php?akt modul=4

[4] Crouzet millennium 3 PLC manual- 2014

[5] Programmable Logic Controllers, W.Bolton, Elsevier-Newnes, 2009 